\title{
Analysis of bike-sharing Niche based on Niche theory
}

\author{
Huixin Liu, Yandan Huang* \\ School of Management Engineering, Zhengzhou University, No.100 Science Avenue, High-Tech \\ Zone of Zhengzhou, China \\ \{liuhuixin,hydzzlg\}@zzu.edu.cn,@163.com
}

Keywords: Shared bikes; Niche theory; Ecological Niche indicator evaluation system ; Niche overlap

\begin{abstract}
With the arrival of the era of "Internet +", Shared bikes, as a new travel mode, have solved the "last three kilometers" problem of daily travel. From the perspective of Niche theory, this paper constructed the framework of capacity Niche in the bike-sharing industry, and calculated the weighted value, evaluation and Niche overlap of the enterprise capacity Niche index through the data integration analysis of Mobike and its competitors. The results show that Mobike is obviously stronger than OFO in terms of innovation ability index, and the Niche overlap degree of the two which is more than 0.8 is accounting for $87.5 \%$, which indicates that there is no significant difference in the Niche superior resources of the Shared bike industry as a whole.
\end{abstract}

\section{Introduction}

Bike-sharing is a green and healthy mode of transportation that takes full advantage of the urban concept of "low-carbon travel". According to the research on quarterly da-ta release of Q2 China bike-sharing industry in 2017, the scale of bike-sharing industry has reached 3.875 billion with a year-on-year growth of 313\%.It's indicate that the bike-sharing industry is in a sustained period of high growth.

The current situation and existing problems of Bike-sharing industry have been studied. The unstandardized consumer behavior, basic facilities such as bicycle lanes and parking area is not complete, and as a for-profit businesses, the profit pattern of enterprise still to be developed(Zixuan Zhang, Wei Wu, 2017 ). Jiya Hu (2017)believes that Shared bikes belong to a market segment of the sharing economy, which improves the efficiency of goods use and reduces resource waste. Yingying Chen and Ruixi liu (2018)analyzed the credit system of the Shared bicycle industry and found that it had problems such as lack of credit supervision, imperfect credit inform ation sharing mechanism and serious lag in standard construction, finally put forward countermeasures and Suggestions for its credit system construction. Minlian Li (2017) conducted a market research and analysis on the Bike-sharing market, and taken a corporate profitability analysis with Mobike and OFO as examples. The results document that OFO's return on capital is higher than Mobike's, but OFO can only maintain profitability for three years, while Mobike can be profitable for seven years. Lihui Li, Dawei Li et al.(2018) also conducted a market research on the Bike-sharing industry, and results illustrated that the human damage problem of Bike-sharing was quite serious, By adopting legal approaches, setting up penalty system for sharing bicycle deposit, strengthening credit system 
and improving residents' quality, this kind of behavior can be effectively restrained. Above is to share the current situation and profit model of the Bike-sharing industry, there are almost no research on Bike-sharing of Niche, this paper is on the basis of predecessors' research to explore the ecological Niche and ecological degree of overlap of Bike-sharing industry from the perspect-ive of the Niche theory, determine the advantages and disadvantages resource-es, hoping to bring some re-ferences to the development of Bike-sharing indus-try.

The traditional bike rental model is "one to one", namely "one person to one car". In the context of sharing economy, sharing bike mode breaks the bou-ndary of traditional rental mode and establishes the "one to $\mathrm{N}$ " rental model which means one bike is av-ailable to all users and potential users. The " one to $\mathrm{N}$ " rental model(fig.1) is based on the network, as well as supported by information technology, which can realizes information transmission and resource sharing among users through information flow and resource flow. With the characteristics of "utilizing idle resources, improving the use efficiency of goods and reducing waste", it realizes resource integration and redistribution through cloud computing, big data and Internet technology(Huixin Liu and XingChen Liu,2015).

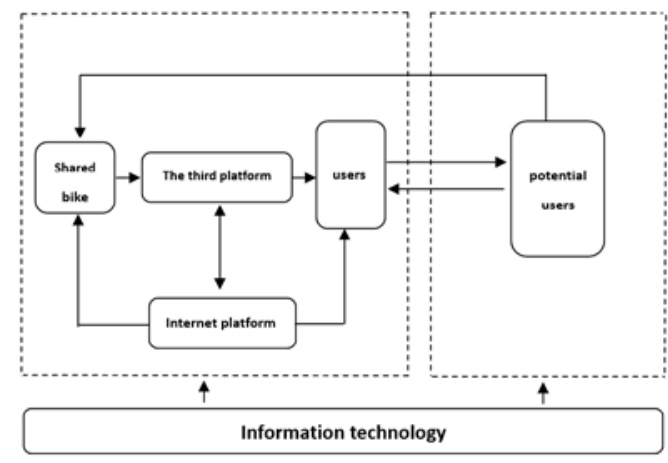

Figure 1 The"one to N" rental model

\section{Constrnction of Capacity Niche Evaluation Framework and Analysis Of Results}

\subsection{Enterprise Capacity Niche Evaluation System}

According to the resource-based theory, enterprises have different intangible and tangible resources that can be transformed into unique capabilities, while the unique resources and capabilities within enterprises are the source of lasting competitive advantages. As a new thing, Shared bike has been popular and loved by netizens once launched, and its market has been filled gradually by the original blank. Due to the gradual development of the Bike-sharing market and the continuous expansion of enterprise resources, the development strategy, organization and marketing ability of the enterprise are very critical for the enterprise to occupy market resources in the initial stage of enterprise development. Therefore, it is more practical to measure the enterprise's ability than resources. According to the "resource-capability-status" enterprise Niche evaluation system(Huixin Liu and XingChen Liu,2015;Xiaoyang Zeng and Jingsong Li 2002), our paper focuses on the establishment of enterprise competence Niche evaluation system from the level of competence, and analyzes the Shared bike enterprises.

\subsection{Evaluation Index and Weight Design of Enterprise Capacity Ecological Niche}

According to the characteristics of Shared bike enterprises, this paper subdivided the ability level. The evaluation of enterprise capability is measured from six aspects: innovation ability, 
organizational ability, supply chain management ability, financial management ability, marketing ability and information system ability.

(1) Innovation ability. The innovation ability is divided into the innovator input, the input of innovation funds and the number of invention patents. Innovation is the first productive force of an enterprise, and also the first weapon for the enterprise to face a competitive environment. Therefore, innovation ability has a high weight. In order to turn scientific and technological resources into actual productive forces through such activities as research and development and technological innovation, the sufficient, stable and high-quality human input in research and development is necessary. Therefore, the innovator input takes up a heavy weight.

(2) Organizing ability. The three dimensions of organizing ability are employee's mentality, ability and management. Therefore, it is measured from three aspects: the strength of enterprise culture cohesion, the work efficiency of employees and the degree of organizational system perfection. The sound degree of the organizational system reflects the overall ability of the enterprise, and the formation of the corporate culture and the work efficiency of employees are based on it. Therefore, the sound degree of the organizational system is highly weighted.

(3) Supply chain management ability. Supply chain mainly includes suppliers, enterprises and customers, so this capability is mainly measured from two aspects: bicycle supplier and quality management capability. Among them, the quantitative index of bicycle suppliers is the rate of superior quality and on-time delivery of the purchased products. These two indicators are both hard and fast indicators to test the capability of suppliers, so the weights are equally divided. Quality management ability starts from two aspects: excellent rate of bicycle quality and rate of bicycle maintenance. With a high rate of excellent bicycle delivery, the maintenance cost of the enterprise will decrease, so the weight of this index is high.

(4) Financial management ability. It can be divided into profitability, debt paying ability and financing ability. Most enterprises aim to make profits. Therefore, profitability is the measure of the success of a business. It is divided into the net interest rate of cost and the rate of return on assets. Debt paying ability is divided into asset-liability ratio and liquidity ratio. The weights of these indicators are evenly divided. The enterprise financing ability is measured from the enterprise financing total amount.

(5) Marketing ability. It's make up of brand management, customer service ability and market share. The brand represents the enterprise's image and appeal which is the enterprise's powerful intangible assets, and the customer service ability is a kind of increment to the enterprise's brand image. Therefore, the weight of customer service ability is relatively high, and its segmentation index, user satisfaction, is a hard index to measure customer service ability which weight is also relatively high.

(6) Information system ability. The information system consists of internal information system and external information system, so the information system capability can be divided into system performance and APP client frequency. The system performance is made up of system smoothness and system security. The smoothness of system performance is the most intuitive feeling of customers for its system, so it takes a large weight.

Here is the weighting of the overall indicator (fig. 2).

\subsubsection{Description of Index Classification and Assignment}

The capability Niche analysis framework used in this paper is divided into four layers, and the degree of subdivision is deepened from left to right. These indicators are divided into quantitative indicators and evaluation indicators.

(1) Quantitative indicators. C1 to C2 elaboration layer (D1, D2, D3), C7 to C12 elaboration layer (D4 to D14), and D16, C3, C14, C16, a total of 18 indicators. These indicators need specific data 
support. For example, the number of patents of Mobike is 32, and the number of OFO patents is 4, Mobike is 10 points, and OFO is $10^{*}(4 / 32)=1.3$ points on the C3 indicator. As of July 2017, the amount of Mobike financing was around $\$ 1$ billion, while the amount of OFO financing was nearly $\$ 1.3$ billion. Therefore, in the index of D12, OFO score was 10 , while Mobike score was $10 *(10 / 13)$ =7.8. $\mathrm{C}$

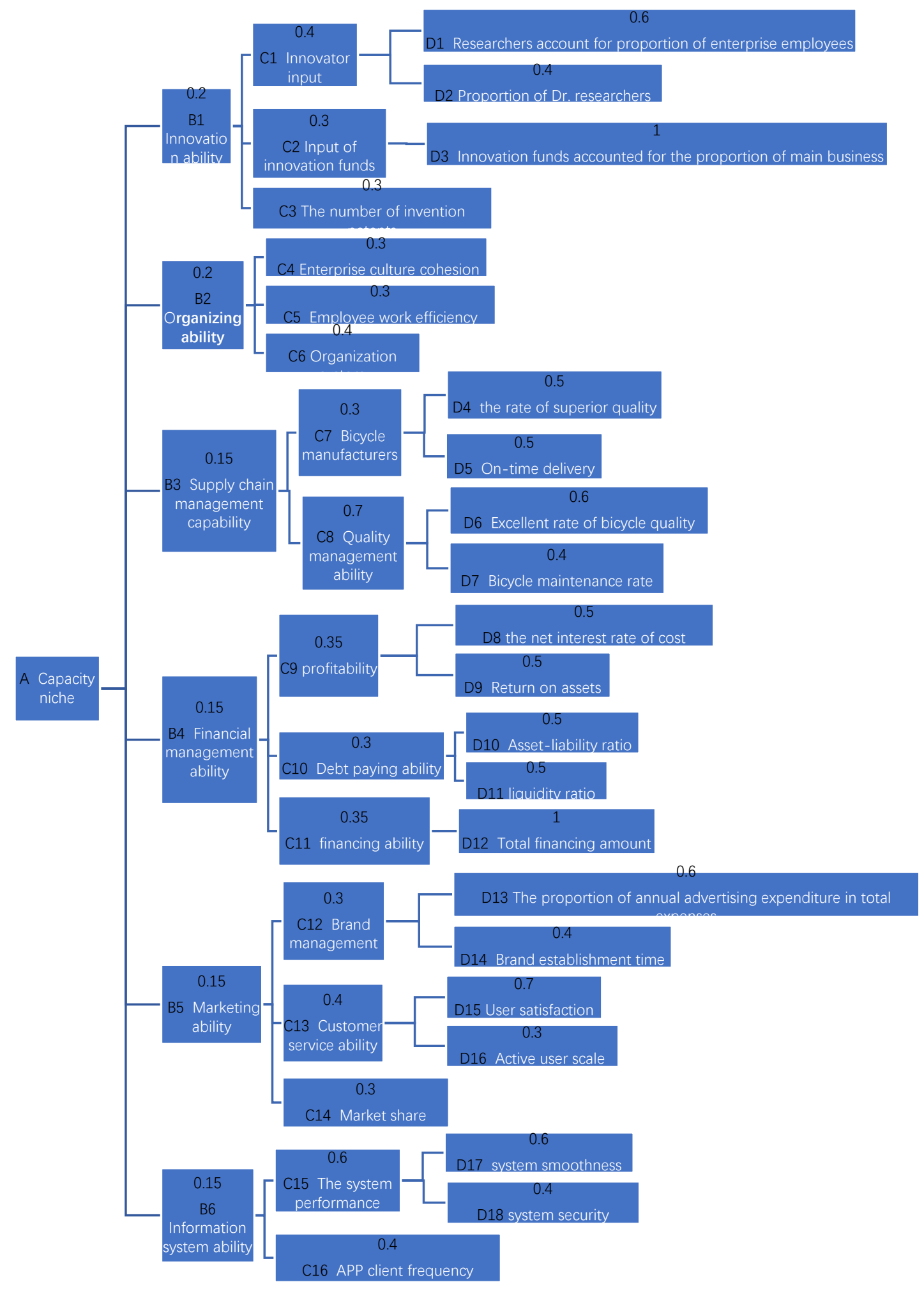

Figure 2 The "resource-capability-status" enterprise Niche evaluation system 
(2) Evaluation indicators. There are 6 indicators in C4, C5, C6, D15, D17 and D18. These indicators are quantified according to specific measurement standards and actual market conditions. For example, according to the user to Shared bike performance experience, such as OFO: vehicle appearance is fresh, and the ride comfort, and high performance/price ratio, while the body of Mobike's bike is designed heavy, and its vehicle standards are higher than OFO two times, the deposit Mobike comprehensive user satisfaction is $67.9 \%$, OFO is $72 \%$. Finally, in D14 user satisfaction index, OFO is 10 points, Mobike for $10 *(67.9 \% / 72 \%)=9.4$. Such quantification has some defects, but it can reflect the actual situation to a certain extent.

\subsection{Enterprise Niche Breadth}

Niche breadth refers to the distance on all possible resources, capabilities and status of all ecological factors dimensions owned by an enterprise, namely the weighted average distance and comprehensive weighted average distance on all ecological factors dimensions of resources, abilities and status (Qi Li, Jinzhao Zhu et al. (2003); Wenhua Li and Furong Han (2006)). According to Wenhua Li's (2006) research, let $L$ be the comprehensive weighted average distance, and $R i$ is the weighted average distance on the dimension of $i$ ecological factor, then

$$
L=\sum_{i=1}^{n}\left(R_{i} * W_{i}\right)
$$

Where $i$ represents the ecological factors of resources, capacity and status, and Wi represents the weight of each ecological factor. The larger the value $L$ is, the stronger the comprehensive competitiveness of enterprise have, and on the contrary, the weaker.

\subsection{Enterprise Niche Breadth Overlap Measurement Method}

Niche overlap means two encounter frequencies at the same resource level (Hurlbert,1978). Huixin Liu, Xingchen Liu (2016) calculated the Niche overlap from the perspective of Niche width.

$L_{i p}$ denotes the occupation width of the ith enterprise on the enterprise capability dimension $p$, $l_{i j p}$ denotes the overlapping width of enterprise $i$ and enterprise $j$ on $p$, then the one-dimensional overlapping degree coefficient of market resource enterprise Niche of enterprise $i$ and enterprise $j$ on $p$ is:

$$
\mathrm{O}_{\mathrm{ij}}=\mathrm{l}_{\mathrm{i}, \mathrm{j}, \mathrm{p}} / / \mathrm{L}_{\mathrm{ip}}
$$

In Equation 2, the $O_{i j}$ represents the ecological Niche overlap coefficient of enterprise $i$ and enter-prise $j$, and $0 \leq O_{i j} \leq 1$. When $O_{i j}=0$, the enterprise Niche overlap degree is 0 which means the two enterprise Niches are completely separated. At this point, there is no competitive relationship between the two enterprises. When $O_{i j}=1$, it can be two situations: when $O_{i j}=O_{j i}=1$, the two enterprises are completely overlapped. In the case of splitting $O_{i j}=1$ and $O_{i j}=0$, the width utilization of this dimension is the relation between include and being include (Huixin Liu and Xingchen Liu (2016); Xingyuan Wang (2006); Jinchao Zhu and Qingke Zhu (2003); Yuyuan Shan et al. (2006)).

\subsection{Frame Assignment and Result Analysis}

According to relevant information of bike-sharing enterprises and IRESEARCH consulting quarterly research report of bike-sharing industry in 2017, the enterprise's ability ecological Niche index is finally assigned with a range of $0-10$. The specific assign-ment is shown in the Table 1.

Combined with figure 2 and Table 1, the ecological Niche results of layer B and layer A can be 
calculated layer by layer from right to left. As shown in Table 2.

Table 1 Final enterprise capability assignment table.

\begin{tabular}{|c|cc|}
\hline $\begin{array}{c}\text { enterprise } \\
\text { Index }\end{array}$ & Mobike & OFO \\
\hline D1 & 10 & 7.8 \\
\hline D2 & 10 & 8.3 \\
\hline D3 & 10 & 7.5 \\
\hline D4 & 8.9 & 10 \\
\hline D5 & 10 & 8.8 \\
\hline D6 & 10 & 8.6 \\
\hline D7 & 10 & 8.9 \\
\hline D8 & 7.8 & 10 \\
\hline D9 & 8.6 & 10 \\
\hline D10 & 10 & 8.7 \\
\hline D11 & 8.7 & 10 \\
\hline D12 & 7.8 & 10 \\
\hline D13 & 5.6 & 10 \\
\hline D14 & 3.3 & 10 \\
\hline D15 & 9.4 & 10 \\
\hline D16 & 10 & 8.4 \\
\hline D17 & 9.6 & 10 \\
\hline D18 & 10 & 9.7 \\
\hline C3 & 10 & 1.3 \\
\hline C4 & 10 & 8.9 \\
\hline C5 & 10 & 9.4 \\
\hline C6 & 8.9 & 10 \\
\hline C14 & 7.2 & 10 \\
\hline C16 & 10 & 9.4 \\
\hline & & \\
\hline & & \\
\hline & \\
\hline & \\
\hline & & \\
\hline & 10.9 \\
\hline
\end{tabular}

Table 2 Evaluation results of enterprise capacity ecological Niche.

\begin{tabular}{|c|c|cc|}
\hline \multicolumn{2}{|c|}{$\begin{array}{c}\text { Enterprise } \\
\text { Ecological Niche }\end{array}$} & Mobike & OFO \\
\hline & Innovation ability & 10 & 5.840 \\
\cline { 2 - 4 } & Organizing ability & 9.560 & 9.490 \\
\cline { 2 - 4 } Layer B & Supply chain management ability & 9.853 & 8.924 \\
\cline { 2 - 4 } & Financial management ability & 8.405 & 9.805 \\
\cline { 2 - 4 } & Marketing ability & 7.396 & 9.808 \\
\cline { 2 - 4 } & Information system ability & 9.826 & 9.688 \\
\hline Layer A & Capacity Niche & 9.236 & 8.800 \\
\hline
\end{tabular}

We can see from the table, Mobike has obvious advantages over OFO in terms of innovation ability. Its organizational ability, supply chain management ability and information system ability score are also higher than OFO, but the differences are not obvious. In terms of financial management ability and marketing ability, it is weaker than OFO. Therefore, through comparison and analysis, Mobike needs to strengthen its marketing and financial management ability. Although its organizational ability is higher than OFO score, the scores of both enterprises are not satisfactory. 
Therefore, it is necessary to form its own unique corporate culture as soon as possible as well as establish and improve corporate rules and regulations to improve the work efficiency of employees.

\subsection{Calculation and Analysis of Enterprise Capacity Niche Overlap}

\subsubsection{Enterprise Capacity Niche Overlap Calculation}

According to the segmentation of enterprise competence Niche and relevant data obtained in figure 2, the excellent enterprise competence is analyzed from the perspective of Niche overlap. Due to the complexity of $\mathrm{D}$ layer segmentation index, this paper only calculated $\mathrm{C}$ layer as the intermediate layer index.

The calculation results are illustrating in Table 3.

Table 3 Index Niche overlap coefficient of layer C

\begin{tabular}{|c|c|}
\hline Enterprise index & Mobike/OFO \\
\hline C1 & 0.8 \\
\hline C2 & 0.75 \\
\hline C3 & 0.13 \\
\hline C4 & 0.89 \\
\hline C5 & 0.94 \\
\hline C6 & 1 \\
\hline C7 & 0.99 \\
\hline C8 & 0.872 \\
\hline C9 $10 ~$ & 1 \\
\hline C11 & 1 \\
\hline C12 & 1 \\
\hline C13 & 1 \\
\hline C14 & 0.994 \\
\hline C15 & 1 \\
\hline C16 & 1 \\
\hline & 0.94 \\
\hline
\end{tabular}

\subsubsection{Results Analysis}

As can be seen from Table 3, the overlapping coefficient of C3 patent quantity index is 0.13 , indicating that Mobike has a big advantage in creativity. Other indicators are above 0.75, it is clearly that Mobike 's overall advantage in the capacity Niche is not apparent when compared with $\mathrm{OFO}$, and this is mainly caused by the Shared bicycle market is nearly two years of emerging markets, the imperfection of the development, and share the bike enter-prises is still in exploring stage, not form a good development model and organizational structure, so the gap on the each ability is narrow.

To sum up, the innovation and organization ability of the company will be the main focus of the future development of the bike-sharing company. By continuously upgrading and iterating products, the company can meet the constantly changing market and intelligent user demand, and at the same time, the company can strengthen the construction of its own culture, organizational structure, rules and regulations, change the profit model and share bicycle enterprises for better development. 


\subsubsection{Results Analysis}

As can be seen from Table 3, the overlapping coefficient of C3 patent quantity index is 0.13 , indicating that Mobike has a big advantage in creativity. Other indicators are above 0.75, it is clearly that Mobike 's overall advantage in the capacity Niche is not apparent when compared with OFO, and this is mainly caused by the Shared bicycle market is nearly two years of emerging markets, the imperfection of the development, and share the bike enterprises is still in exploring stage, not form a good development model and organizational structure, so the gap on the each ability is narrow.

To sum up, the innovation and organization ability of the company will be the main focus of the future development of the bike-sharing company. By continuously upgrading and iterating products, the company can meet the constantly changing market and intelligent user demand, and at the same time, the company can strengthen the construction of its own culture, organizational structure, rules and regulations, change the profit model and share bicycle enterprises for better development.

\section{Conclusions}

Under the "one to N" rental model, the Shared bike enterprises can quickly occupy market share and seize market resources through the Internet platform. They can also form a service circle with other third-party service enterprises and expand the service scope to increase the user scale. Under the influence of the Internet, the number of enterprise users will increase exponentially and the operating revenue of the Shared bike enterprises will increase with the increase of users, but the marginal cost of each bike put on the market will decrease. In this process, the enterprise's own ability plays a decisive role in the expansion of the market network, and its marketing ability and supply chain management ability are the necessary skills to increase the user scale. According to the capacity Niche analysis of Mobike and OFO, it can be seen that OFO is better in marketing ability and has obvious advantages in user quantity and scale. Through the analysis of the bike-sharing enterprise's ability Niche and overlapping degree, it can be seen that the development of bike-sharing industry at the present stage will be a competition between the enterprise's innovation ability and the user scale. This paper only analyzes the capacity Niche of the bike-sharing industry, which is the deficiency of this paper, but it still hopes to be helpful to the bike-sharing industry.

\section{References}

[1] Huixin Liu ,Xingchen Liu.2015. The analysis of enterprise resources based on the ecological Niche evaluation system of "resource-capacity-position", Enterprise Economy. (1), pp.11-16.

[2] Hurlbert S H.1978.The measurement of Niche overlap and some relatives, Ecology.59 (1), pp. 67-77.

[3] Huixin Liu, Xingchen Liu.2016. Study on resources allocation of ShunFeng commercial ecosystem, academic dissertation of Zhengzhou University.

[4] Jiya Hu. 2017. Sharing economy and sharing bike, Journal of Chinese Youth Social Science.36 (5), pp.8-10.

[5] Jinzhao Zhu, Qingke Zhu.2003. Advances in ecological Niche theory and its measurement, Journal of Beijing forestry university.(1), pp.100-107.

[6] Lihui Li, Dawei Li ,Weiji Li.2018. Research on the damage problem of Shared bike and countermeasures, Social Science in Hunan.(2), pp.116-120.

[7] Minlian Li.2017. Share bike market research and analysis,Money China.(5), pp.121-123.

[8] Wenhua Li, Furong Han.2006. Study on measurement method of enterprise ecological Niche parameter, Journal of Beijing university of technology.(4), pp.379-384.

[9] Xiaoyang Zeng,Jingsong Li.2002. On the construction of evaluation index system of marketing competitiveness, Journal of Commercial economy and management.(5), pp.9-13.

[10] Xingyuan Wang.2006. Research on the calculation model of Niche overlap degree of similar brand market, China marketing association 2006 annual meeting and the fourth national congress of the symposium. 
[11] Yuyuan Shan, Guo Li, Dan Chen.2006. Research on Enterprises' Competitive Strategies Based on Ecology Niche Theory, Science of Science and Management of S.\& T.27(3), pp.159-163.

[12] Zixuan Zhang, Wei Wu.2017. Current situation, problems and development countermeasures of Shared bikes, Modern Business.(15), pp.162-163. 